\title{
GW23-e1525 THE COMPLEMENT C10 TUMOUR NECROSIS FACTOR-RELATED PROTEIN 1 LEVEL IN PATIENTS WITH SLEEP APNOEA AND PRIMARY ALDOSTERONISM
}

doi:10.1136/heartjnl-2012-302920a.273

Yao Xiao-guang, Cheng Wei-ping, Ma Wan-yong, Yan Zhi-tao, Bi Yun-wei, Li Nanfang. Hypertension Institute of Xinjiang

Objectives To discuss the serum level of complement c1q tumour necrosis factor-related protein 1 (CTRP1) and its related factors in patients with sleep apnoea obstructive sleep apnoea (OSA) and primary aldosteronism (PA).

Methods A total of 28 in patients with confirmed OSA and PA were selected. As controls, 30 cases with OSA and essential hypertensive were matched for apnoea hypopnoea index (AHI) and plasma rennin activity (PRA). The levels of PRA, angiotensin II (AngII) and aldosterone (Ald) were measured by adioimmunodetection and the level of serum CTRP1 were determined by ELISA in all subjects.

Results The level of Ald was significantly higher in patients with OSA and PA than that in controls $(p=0.037)$, but no significant difference in the levels CTRP1, pRA, AngII, C-reaction protein (CRP), serum potassium and sodium were detected between two groups. Pearson correlation analysis indicated no correlation between the level of CTRP1 and the levels of ALD, AHI in the whole population but negative correlation with AngII $(r=-0.454$, $\mathrm{p}<0.05$ ) in patients with OSA with PA.

Conclusions No difference of the level of CTRP1 was detected between patients with OSA and PA and patients with OSA and essential hypertension. It is the level of AngII rather than aldosterone negatively related to the level of CTRP1 in patients with OSA and PA. 